\title{
Article \\ Effect of Different Water Quality on the Nutritive Value and Chemical Composition of Sorghum bicolor Payenne in Cape Verde
}

\author{
Vanessa Mendoza-Grimón ${ }^{1}$ (D), Regla Amorós ${ }^{2}$, Juan Ramón Fernández-Vera ${ }^{3}$, Jose Manuel Hernádez-Moreno ${ }^{1}$ \\ and María del Pino Palacios-Díaz ${ }^{1, * \mathbb{D}}$ \\ 1 Instituto de Investigación IUNAT, Grupo GEOVOL, Universidad de Las Palmas de Gran Canaria, \\ 35001 Las Palmas de Gran Canaria, Spain; v.mendozagrimon@ulpgc.es (V.M.-G.); \\ jhmoreno@ull.es (J.M.H.-M.) \\ 2 Instituto Nacional de Investigação e Desenvolvimento Agrário (Inida), São Jorge dos Orgaos, Assomada, \\ Santiago, Cidade da Praia 84, Cape Verde; regla.amoros@inida.gov.cv \\ 3 Laboratorio Agroalimentario y Fitopatológico del Cabildo de Gran Canaria, 35013 Arucas, Spain; \\ jrfernandezv@grancanaria.com \\ * Correspondence: mp.palaciosdiaz@ulpgc.es; Tel.: +34-928-454-353; Fax: +35-928-451-142
}

check for updates

Citation: Mendoza-Grimón, V.; Amorós, R.; Fernández-Vera, J.R.; Hernádez-Moreno, J.M.; Palacios-Díaz, M.d.P. Effect of Different Water Quality on the Nutritive Value and Chemical Composition of Sorghum bicolor Payenne in Cape Verde. Agronomy 2021, 11, 1091. https://doi.org/ 10.3390/agronomy11061091

Academic Editors:

Dominika Średnicka-Tober and Ewelina Hallmann

Received: 15 April 2021

Accepted: 25 May 2021

Published: 28 May 2021

Publisher's Note: MDPI stays neutral with regard to jurisdictional claims in published maps and institutional affiliations.

Copyright: (c) 2021 by the authors. Licensee MDPI, Basel, Switzerland. This article is an open access article distributed under the terms and conditions of the Creative Commons Attribution (CC BY) license (https:// creativecommons.org/licenses/by/ $4.0 /)$.

\begin{abstract}
Cape Verde is a semiarid country where lack of rainfall exacerbates the scarce resources available for livestock which, therefore, make it very vulnerable to climate change. By providing reclaimed water (RW) for irrigation, it is possible to decrease forage importation. Subsurface drip irrigation (SDI) improves health security by preventing contact between water and harvested plants. Sorghum is a water-efficient crop that provides good nutritional value. The aim of this experiment was to study the nutrient and fiber contents of the Sorghum Payenne variety using subsurface (T1) and surface (T2) drip irrigation by RW vs. conventional water (T3) and plant maturity to assure the feasibility of water reuse to produce forage. Ntot-Ptot-Ca-Mg and Na were significantly higher in the RW plants than in the conventional water ones. Ntot-Ptot-K and Fe contents significantly lowered, while $\mathrm{Ca}-\mathrm{Na}$ and $\mathrm{Mn}$ significantly rose as plant maturity increased. All the fiber values meet the Nos. 2 and 3 quality standards, and the Prime and No. 1 for NDF and ADF, respectively. The obtained good forage quality let to avoid the competence of conventional water and to reuse nutrients added by RW. If generalized, this solution would reduce forage importation by improving food sovereignty and farmers' profitability, and would enhance resilience against climate change effects.
\end{abstract}

Keywords: minerals; NDF; ADF; SDI; treated water reuse; water management; food sovereignty; climate change

\section{Introduction}

Animal production plays a crucial role in rural economy and food sovereignty. Water scarcity limits livestock development in semiarid regions as high water prices represent an elevated percentage of total forage production costs [1]. In fact, animal feeding largely depends on imported forages in these areas, which poses economic and strategic problems for farmers [2] because, under rain-fed conditions, fodder resources are not evenly available all year long. Conversely, forage production using irrigation can be predictable, and can avoid the dependence of feeding importation if profitable. Besides, forage production is the most suitable choice for reusing Reclaimed Water (RW) given its lower quality demands and lack of fresh forage crops to feed livestock in arid regions [3]. Thus, by providing water for irrigation purposes, these resources lead to fewer imports of food supplies [4]. To ensure sanitary safety when reusing RW, Subsurface Drip Irrigation (SDI) is one of the best options because it requires lower water treatment than any other alternative, and improves health security by preventing contact between water and stems and leaves which, thus, 
minimizes sanitary risks [5]. Besides, SDI systems reduce the amount of water compared to water estimated by ETc, as practically all the supplied water is absorbed by plants [4].

Cape Verde exemplifies the above-mentioned semiarid country where lack of rainfall exacerbates scarce resources for livestock. Its farming, pastoralism, and forestry are very vulnerable because of poor natural resources, an arid climate and the prevalence of traditional low-intensive farming systems. Agricultural production is based on rain-fed systems, and only 3500 hectares of the total agricultural area (79,000 ha) are irrigated [6]. Evacuation of wastewater through the public sewage system and septic tank represents a percent of $74 \%$ in Cape Verde, which has a percentage of $21.0 \%$ of households connected to the public sewage system, $31.0 \%$ in urban areas and $1.3 \%$ in rural areas. Santiago island has five of the total of eight installed Waste Water Treatment Plants (WWTP). On this island, four of these WWTP are connected to rural villages, next to agricultural areas, and provide $1475 \mathrm{~m}^{3}$ /day of treated water [7].

This country very much depends on imports for food supplies and it imports more than $80 \%$ of its food. According to the 2015 Agricultural Census, the agricultural structure comprises 45,399 farms, an active agricultural population of 92,322 people, almost all farms are family-owned. The majority of farm parcels are on Santiago island (59\%), livestock is practiced on $85.3 \%$ of farms, while dry farming is practiced on $73.4 \%$ of these parcels. Given the drought in Cape Verde, the reduction in animal herds (especially cattle and goats) is drastic [8]. A decrease in the number of goats and pigs by $37.6 \%$ and $15.5 \%$, respectively, and an increase in cows $(24.6 \%)$ and sheep (17.5\%) are calculated using data from 2004 to 2015. Family livestock farms are an important component of income and livelihoods of farming families, and the most frequently raised species are chickens $(75.4 \%)$ and goats $(12.2 \%)$ [9], whose feed depends on forage imports. However, sorghum is very droughttolerant compared to other forage crops [10], more water-efficient than corn [11] and is able to produce higher dry matter yields and nutrition values than maize [12,13]. Some authors have indicated that milk production from dairy cattle and weight gain obtained from feeding cattle sorghum have been shown to equal those fed maize [14,15].

There are several Sorghum bicolor L. Moench hybrids and varieties with different nutrition values [16], whose values need to be determined to design proper animal feed. There is not enough information available about the nutritional values of the Payenne variety, which is a cross between CE 151-262 X Sarvato-1. Its principal agronomic characteristics are: a sowing-flowering cycle of 60-63 days; a seedling-maturity cycle of 87-90 days; not photosensitive; barely resists lodging; sensitive in humid areas; grain weight (g) of 25.09 (1000 grains); maximum yield of $3500 \mathrm{~kg} / \mathrm{ha}$ [17].

Some important nutrition properties of fodder crops are fiber and macro- and micronutrient contents. Fiber concentration increases, while quality and digestibility decrease as aging prolongs. Acid detergent fiber (ADF), acid detergent lignin (ADL), and neutral detergent fiber (NDF) are widespread as standard forage testing techniques for fiber analyses. ADF can be used to calculate digestibility, while the intake potential is predicted with NDF [18]. Assessing the nutritional value of sorghum varieties would have a direct impact on improving Sorghum for quality breeding and for developing food products [19].

The aim of this experiment was to study the chemical/nutritional characteristics of the Sorghum bicolor Payenne variety that is drip-irrigated using two water qualities in Cape Verde in different plant maturity stages. As far as we know, no information on the nutritional behavior of this variety is available, which is an added value of this study. This research study works toward the possibility of irrigation reuse with treated water to produce on-farm fodder that covers the nutrient requirements of animals in Cape Verde by partially replacing forage importation.

\section{Materials and Methods}

\subsection{Experimental Field}

In 2019, a Sorghum experiment was conducted in a field $\left(540 \mathrm{~m}^{2}\right)$ located in Rocha Lama (15 $7^{\prime} 43^{\prime \prime}$ N; $23^{\circ} 31^{\prime} 38^{\prime \prime}$ W. 6 asl), Santa Cruz on Santiago Island, Cape Verde. The 
area has a warm, humid, and sunny climate, with a mean minimum temperature, a mean maximum temperature, a mean humidity, and hours of sunlight of $21.4^{\circ} \mathrm{C}, 28.9{ }^{\circ} \mathrm{C}, 70 \%$, and $7.2 \mathrm{~h}$, respectively [4].

After preliminary works, which consisted of removing stones and plowing in $950 \mathrm{~kg}$ of cow's manure, the Sorghum bicolor Payenne variety was seeded: holes spaced $0.2 \mathrm{~m}$ with 4 seeds per hole on 8 April 2019. Plants were harvested between the soft and hard dough stages. Four harvests were achieved: the first productive period lasting 90 days (from 8 April 2019 to 8 July 2019); a second 53-day period (8 July 2019 to 29 August 2019); a third 100-day period (29 August 2019 to 17 December 2019); and last harvest lasting 48 days (until February 2020). Hence, higher mature plants were harvested in the first and third harvest while less matured in the second and fourth ones. No rainfall fell during the experimental period. No chemical weed or pest control was used and emerging weeds were controlled by hand hoeing.

The experimental field was irrigated using three treatments based on water quality (conventional vs. RW) and drip system (subsurface drip, SDI vs. surface drip SFDI): T1: RW applied by SDI $\left(60 \mathrm{~m}^{2}\right)$; T2: RW plus SFDI $\left(60 \mathrm{~m}^{2}\right)$; T3: conventional water plus SDI $\left(60 \mathrm{~m}^{2}\right)$.

These areas were repeated in three blocks in which all three treatments were irrigated (9 plots). Each plot consisted of eight lines separated $0.75 \mathrm{~m}$ that were $10 \mathrm{~m}$ long. Each irrigation treatment applied the estimated water use according to CROPWAT using the weather station data from Praia [20]. The same water volume was applied for both SDI and SFDI, with a total water quantity equivalent to $11,044 \mathrm{~m}^{3} /$ ha for the whole study period.

\subsection{Irrigation System}

An irrigation head with one controller and two different lines, each consisting in one pump and one sand filtration system for each water quality, were installed. Besides, a UV disinfection lamp was installed to the applied RW. Integral drippers operating at delivery rates of $2.3 \mathrm{~L} / \mathrm{h}$ were employed. Lateral lines were spaced $0.75 \mathrm{~m}$ and buried at a depth of $0.20 \mathrm{~m}$. Irrigation was provided twice daily. Each treatment had its own flow meter.

\subsection{Water Quality}

Sta Cruz WWTP, which presents low-energy consumption and is adapted to other rural villages in Cape Verde, supplied the RW employed in this experiment in T1 and T2. The treatment system consists of a pretreatment area, an anaerobic digester as the primary treatment, and a series of vertical flow gravel filtration beds as the secondary treatment. The designed total water treatment capacity was $1000 \mathrm{~m}^{3}$ per day. Recently, this plant effectively treats $200 \mathrm{~m}^{3}$ per day [7]. The treated water characteristics analyzed by INLAB using internal method based on Decree-Law 236 of 1998 of Portugal, are shown in Table 1. Table 2 offers the groundwater quality from wells close to the experimental field (wells PT33 and FT59), and were used as conventional water (T3). The groundwater samples were analyzed by the Laboratorio Agroalimentario del Cabildo de Gran Canaria: $\mathrm{pH}$ and EC were analyzed by an electrometric method soon after sample collection. Water samples were filtered through a $0.45-\mu \mathrm{m}$ pore membrane filter soon after sample collection. For metal and metalloid analysis, a filtered aliquot sample was acid stabilized $(\mathrm{pH}<2)$ and stored at $4{ }^{\circ} \mathrm{C}$ until analysis by Atomic Emission Spectroscopy Optical Emission (ICP-OES). A second aliquot, for major anions $\left(\mathrm{Cl}^{-}, \mathrm{NO}_{3}{ }^{-}\right.$, and $\left.\mathrm{SO}_{4}{ }^{2-}\right)$, was stored frozen until ion chromatography analysis. SAR was calculated by equation: $S A R=\frac{[\mathrm{Na}]}{\sqrt{\frac{([\mathrm{Ca}]+[\mathrm{Mg}]}{2}}}$. 
Table 1. Chemical parameters analyzed from the treated water.

\begin{tabular}{|c|c|c|c|c|c|c|c|c|c|c|c|c|}
\hline & & & $\mu \mathrm{S} / \mathrm{cn}$ & & & & & $\mathrm{mg} / \mathrm{L}$ & & & & \\
\hline & & $\mathrm{pH}$ & EC & COD & BOD $_{5}$ & $\mathrm{NO}_{3}{ }^{-}$ & SAR & $\mathrm{Cl}^{-}$ & $\mathbf{N a}$ & $\mathrm{Ca}$ & Mg & TSS \\
\hline \multirow{2}{*}{ RW } & mean & 7.5 & 2970 & 32 & 6.3 & 320 & 6.8 & 415 & 361.6 & 91.3 & 71.4 & 214.0 \\
\hline & std & 0.7 & 355.9 & 1.4 & 0.4 & 157.2 & 2.1 & 35.4 & 91.3 & 5.5 & 9.9 & 19.8 \\
\hline
\end{tabular}

Table 2. Chemical parameters analyzed of the groundwater from two wells.

\begin{tabular}{|c|c|c|c|c|c|c|c|c|c|c|c|c|c|c|c|c|}
\hline & & & $\mu \mathrm{S} / \mathrm{cn}$ & (meq/) & )$^{1 / 2}$ & & & & & & $\mathrm{ng} / \mathrm{L}$ & & & & & \\
\hline Wells & & $\mathrm{pH}$ & EC & SAR & $\mathrm{Na}$ & $\mathbf{K}$ & $\mathrm{Ca}$ & Mg & $\mathrm{Cl}^{-}$ & $\mathrm{NO}_{3}{ }^{-}$ & $\mathrm{SO}_{4}^{2-}$ & B & $\mathrm{Cu}$ & $\mathrm{Fe}$ & $\mathrm{Zn}$ & Mn \\
\hline \multirow{2}{*}{ PT33 } & mean & 8.1 & 1150 & 2.2 & 99 & 9.15 & 55 & 62 & 140 & 45.5 & 39.5 & 0.135 & $<0.015$ & $<0.015$ & 0.016 & $<0.005$ \\
\hline & std & 0 & 50 & 0.03 & 1 & 0.05 & 1 & 2 & 0 & 1.5 & 2.5 & 0.005 & - & - & - & - \\
\hline \multirow{2}{*}{ FT59 } & mean & 7.95 & 1250 & 1.285 & 65.5 & 7.3 & 96 & 60.5 & 190 & 45 & 46 & 0.07 & $<0.015$ & $<0.015$ & $<0.010$ & $<0.005$ \\
\hline & std & 0 & 106.1 & 0.01 & 2.47 & 0.28 & 9.90 & 5.30 & 42.42 & 0.71 & 1.41 & 0 & - & - & - & - \\
\hline
\end{tabular}

\subsection{Soil Analysis}

The soils of the experimental field have been modified by human activity and have lost their horizons. According to the Soil Taxonomy [21], soils belong to the suborder of Arents: Torriarents isoperthermic. According to the FAO classification [22], they would be considered in the Anthrosols Group, with the qualifier "irragric" and, after taking into account the salinity achieved during the last sampling, also with the qualifier "salic".

Soil samples were taken from the first $0.2 \mathrm{~m}$ prior to manure application (May 2017), post-manure application (November 2018), and seeding day (April 2019), and coincided with the first harvest. Samples were taken from all the treatments. Organic carbon (OC, $\%$ ) and nitrogen $(\mathrm{N}, \%)$ were determined by dry combustion with a LECO CNS 2000 analyzer. Soluble salts were estimated by electrical conductivity EC1:5 (soil:water ratio; $\mathrm{dS} / \mathrm{m}$ ). Available nitrate was determined by soil extraction, also at the 1:5 ratio, with 0.01 $\mathrm{M}$ calcium chloride, and analyzed by ionic chromatography. Available soil P (mg/kg) was determined by sodium bicarbonate extraction according to the method of Olsen [23]. Exchangeable cations $(\mathrm{K}, \mathrm{Ca}, \mathrm{Mg}$, and $\mathrm{Na}$, meq/100 g) were extracted with buffered $1 \mathrm{M}$ ammonium acetate at $\mathrm{pH}$ 7. Microelements $\mathrm{B}, \mathrm{Fe}, \mathrm{Cu}, \mathrm{Mn}$, and $\mathrm{Zn}(\mathrm{mg} / \mathrm{kg})$ were extracted with DTPA pH 7. Both cations and microelements were analyzed by ICP. All the parameters were determined at the Laboratorio Agroalimentario del Cabildo de Gran Canaria.

\subsection{Forage Characterization}

Forage characterization: one linear meter from three rows was randomly sampled from each plot (three treatments $\times$ three blocks) at all four harvests to characterize the nutritive value of the Sorghum per treatment. This study quantified: $\mathrm{N}$, as determined by dry combustion in LECO CNS 2000; macro- and microelements ( $\mathrm{P}, \mathrm{K}, \mathrm{Ca}, \mathrm{Mg}, \mathrm{Na}, \mathrm{B}$, $\mathrm{Cu}, \mathrm{Fe}, \mathrm{Mn}$, and $\mathrm{Zn}$ ) using digestion with nitric acid in a microwave and analyzed by inductively coupled plasma optical emission spectrometry (ICP_OES); NDF and ADF contents, analyzed following the Van Soest methodology [24].

\subsection{Statistical Analysis}

Analyses of variance (MANOVA for the plant parameters) were carried out using the SPSS statistical package (version 27) by the Generalized Linear Model. The model included treatment, harvest (plant maturity: a bigger number of days between harvests) and their interactions. The interaction was not significantly different. Levene's test of equality of error variances was used for analyzing the experimental data. Only the first and third harvests showed that the error variance of NDF was not equal across groups. The variances for the means of $\mathrm{N}, \mathrm{Mn}, \mathrm{Zn} \mathrm{B}$, and for medians of $\mathrm{P}, \mathrm{K}, \mathrm{Ca} \mathrm{Mg} \mathrm{Na}, \mathrm{Cu}$, and $\mathrm{Fe}$, were 
homogeneous. F tests were done based on linearly independent pairwise comparisons among the estimated marginal means. Separation subsets were tested by considering $p=0.05$ using Tukey's and Games-Howell tests for homogenous and non-homogenous variances, respectively.

\section{Results and Discussion}

\subsection{Soil Evolution}

Table 3 presents the soil evolution due to manure incorporation, forage cultivation, irrigation, and water quality used. Only soil $\mathrm{pH}, \mathrm{Ca}, \mathrm{Mg}$, and Fe presented values in the same interval during the whole period. The rest of the parameters experimented increments, soon after the manure addition ( $\mathrm{P}$ and $\mathrm{Mn}$ ) or at seeding time (prior to irrigation): $\mathrm{OM}$, $\mathrm{EC}$, Ntot, $\mathrm{C} / \mathrm{N}$, nitrate, $\mathrm{K}$, and micronutrients $\mathrm{B}, \mathrm{Cu}, \mathrm{Mn}$, and $\mathrm{Zn}$. Na increments seem to be associated with irrigation and not with manure addition. Only $\mathrm{C} / \mathrm{N}$ and $\mathrm{Mn}$ values decreased after the irrigation period, while the rest increased their contents in soil. As pointed out by Palacios-Diaz et al. [25] the increment in microbiological activity, because of the constant water availability, can partially explain this result. In agreement with the mentioned study, a high increment in salinity is observed, more remarkable in T1 than in $\mathrm{T} 2$ or T3. Nitrate capillary rise from treated water of T1 can explain this result, as $\mathrm{Na}$ is similar in T1 and T2, but nitrate is clearly higher in T1. Hence, out of the rain period, no nitrate leaching is expected by this system. In this sense, sorghum, as a $\mathrm{C} 4$ grass able to absorb high nitrate content and salinity tolerant, is an optimal crop. As expected, Ntot and $\mathrm{Na}$ were higher in soils irrigated with RW (T1 and T2) than with well water (T3) while orthophosphate was not, according to Palacios-Diaz et al. [25]. Therefore, in general terms, soil fertility increased in the proposed water reuse system. However, irrigation water management should be optimized to avoid soil salinity buildup.

Table 3. Soil properties evolution among stages: pre- and post-manure, seeding, and post first harvest, for treatments $\mathrm{T} 1$, T2, and T3 (T1: reclaimed water plus subsurface drip; T2: reclaimed water plus surface drip; T3: conventional water plus subsurface drip irrigation), expressed as mean and standard deviation.

\begin{tabular}{|c|c|c|c|c|c|c|c|c|c|c|c|c|c|c|c|c|c|c|}
\hline \multirow[b]{2}{*}{ Stage } & \multirow{2}{*}{\multicolumn{2}{|c|}{ Treat }} & \multirow[b]{2}{*}{$\mathrm{pH}$} & \multirow{2}{*}{$\begin{array}{l}\mathrm{dS} / \mathrm{m} \\
\mathrm{EC} 1: 5\end{array}$} & \multicolumn{2}{|c|}{$\%$} & \multirow[b]{2}{*}{$\mathrm{C} / \mathrm{N}$} & & \multicolumn{4}{|c|}{$\mathrm{meq} / 100 \mathrm{~g}$} & \multicolumn{5}{|c|}{$\mathrm{mg} / \mathrm{kg}$} \\
\hline & & & & & OM & Ntot & & Nitrate & $P$ & K & $\mathrm{Ca}$ & Mg & $\mathrm{Na}$ & B & $\mathrm{Cu}$ & $\mathrm{Fe}$ & Mn & $\mathrm{Zn}$ \\
\hline \multicolumn{2}{|l|}{ pre_manure } & $\begin{array}{c}\text { mean } \\
\text { std }\end{array}$ & $\begin{array}{c}8 \\
0.19\end{array}$ & $\begin{array}{l}0.76 \\
0.19\end{array}$ & $\begin{array}{c}1.3 \\
0.17\end{array}$ & $\begin{array}{c}0.1 \\
0.01\end{array}$ & $\begin{array}{c}8.2 \\
0.55\end{array}$ & $\begin{array}{c}737.3 \\
169.47\end{array}$ & $\begin{array}{l}56.0 \\
3.46\end{array}$ & $\begin{array}{l}3.3 \\
0.35\end{array}$ & $\begin{array}{l}29.1 \\
10.04\end{array}$ & $\begin{array}{l}15.6 \\
2.56\end{array}$ & $\begin{array}{l}6.7 \\
2.95\end{array}$ & $\begin{array}{l}1.1 \\
0.34\end{array}$ & $\begin{array}{l}1.2 \\
0.15\end{array}$ & $\begin{array}{l}5.4 \\
2.00\end{array}$ & $\begin{array}{l}2.3 \\
0.31\end{array}$ & $\begin{array}{l}1.1 \\
0.24\end{array}$ \\
\hline \multicolumn{2}{|l|}{ post_manure } & $\begin{array}{l}\text { mean } \\
\text { std }\end{array}$ & $\begin{array}{c}8.2 \\
0.12\end{array}$ & $\begin{array}{l}0.89 \\
0.39\end{array}$ & $\begin{array}{c}1.8 \\
0.50\end{array}$ & $\begin{array}{l}0.12 \\
0.03\end{array}$ & $\begin{array}{c}8.5 \\
0.68\end{array}$ & $\begin{array}{c}648.3 \\
300.43\end{array}$ & $\begin{array}{l}65.0 \\
9.85\end{array}$ & $\begin{array}{l}3.6 \\
0.85\end{array}$ & $\begin{array}{l}26.5 \\
9.92\end{array}$ & $\begin{array}{l}14.1 \\
2.29\end{array}$ & $\begin{array}{l}5.7 \\
1.39\end{array}$ & $\begin{array}{l}1.4 \\
0.17\end{array}$ & $\begin{array}{l}1.2 \\
0.18\end{array}$ & $\begin{array}{l}5.4 \\
1.07\end{array}$ & $\begin{array}{l}4.4 \\
0.53\end{array}$ & $\begin{array}{l}1.1 \\
0.24\end{array}$ \\
\hline \multirow{3}{*}{ seeding } & $\mathrm{T} 1$ & $\begin{array}{c}\text { mean } \\
\text { std }\end{array}$ & $\begin{array}{c}8 \\
0.14\end{array}$ & $\begin{array}{c}1.6 \\
0.92\end{array}$ & $\begin{array}{c}2.5 \\
0.55\end{array}$ & $\begin{array}{c}0.2 \\
0.03\end{array}$ & $\begin{array}{c}9.2 \\
0.78\end{array}$ & $\begin{array}{c}984.0 \\
473.56\end{array}$ & $\begin{array}{l}87.0 \\
3.46\end{array}$ & $\begin{array}{l}5.9 \\
1.37\end{array}$ & $\begin{array}{l}19.6 \\
1.75\end{array}$ & $\begin{array}{l}15.1 \\
1.32\end{array}$ & $\begin{array}{l}6.9 \\
2.07\end{array}$ & $\begin{array}{l}1.6 \\
0.15\end{array}$ & $\begin{array}{l}1.5 \\
0.74\end{array}$ & $\begin{array}{l}4.7 \\
0.10\end{array}$ & $\begin{array}{l}11.1 \\
0.65\end{array}$ & $\begin{array}{l}1.8 \\
0.41\end{array}$ \\
\hline & $\mathrm{T} 2$ & $\begin{array}{c}\text { mean } \\
\text { std }\end{array}$ & $\begin{array}{c}8.0 \\
0.12\end{array}$ & $\begin{array}{c}2.2 \\
0.70\end{array}$ & $\begin{array}{l}2.46 \\
0.53\end{array}$ & $\begin{array}{c}0.2 \\
0.01\end{array}$ & $\begin{array}{c}8.7 \\
1.61\end{array}$ & $\begin{array}{l}1124.0 \\
437.12\end{array}$ & $\begin{array}{l}82.7 \\
16.77\end{array}$ & $\begin{array}{l}6.0 \\
0.26\end{array}$ & $\begin{array}{l}18.3 \\
0.72\end{array}$ & $\begin{array}{l}13.7 \\
1.99\end{array}$ & $\begin{array}{l}8.1 \\
0.95\end{array}$ & $\begin{array}{l}1.4 \\
0.15\end{array}$ & $\begin{array}{l}1.4 \\
0.00\end{array}$ & $\begin{array}{l}4.4 \\
0.21\end{array}$ & $\begin{array}{l}11.9 \\
1.72\end{array}$ & $\begin{array}{l}1.9 \\
0.13\end{array}$ \\
\hline & T3 & $\begin{array}{c}\text { mean } \\
\text { std }\end{array}$ & $\begin{array}{c}8.0 \\
0.12\end{array}$ & $\begin{array}{l}1.6 \\
0.07\end{array}$ & $\begin{array}{c}3.2 \\
0.82\end{array}$ & $\begin{array}{c}0.2 \\
0.03\end{array}$ & $\begin{array}{l}10.0 \\
1.00\end{array}$ & $\begin{array}{l}842.7 \\
11.85\end{array}$ & $\begin{array}{l}108.0 \\
40.73\end{array}$ & $\begin{array}{l}6.9 \\
1.42\end{array}$ & $\begin{array}{l}25.2 \\
12.24\end{array}$ & $\begin{array}{l}12.9 \\
2.40\end{array}$ & $\begin{array}{l}6.1 \\
1.39\end{array}$ & $\begin{array}{l}1.7 \\
0.25\end{array}$ & $\begin{array}{l}1.4 \\
0.15\end{array}$ & $\begin{array}{l}4.4 \\
0.06\end{array}$ & $\begin{array}{l}15.7 \\
4.58\end{array}$ & $\begin{array}{l}2.0 \\
0.37\end{array}$ \\
\hline \multirow{3}{*}{$\begin{array}{c}\text { Post first } \\
\text { harvest }\end{array}$} & $\mathrm{T} 1$ & $\begin{array}{c}\text { mean } \\
\text { std }\end{array}$ & $\begin{array}{c}8.0 \\
0.05\end{array}$ & $\begin{array}{l}7.1 \\
0.23\end{array}$ & $\begin{array}{c}3.5 \\
0.35\end{array}$ & $\begin{array}{c}0.3 \\
0.03\end{array}$ & $\begin{array}{c}6.3 \\
0.07\end{array}$ & $\begin{array}{l}5175.0 \\
219.20\end{array}$ & $\begin{array}{l}80.5 \\
7.78\end{array}$ & $\begin{array}{l}8.8 \\
0.57\end{array}$ & $\begin{array}{l}21.3 \\
1.48\end{array}$ & $\begin{array}{l}16.0 \\
0.07\end{array}$ & $\begin{array}{l}12.9 \\
2.76\end{array}$ & $\begin{array}{l}2.1 \\
0.14\end{array}$ & $\begin{array}{l}1.7 \\
0.28\end{array}$ & $\begin{array}{l}5.0 \\
0.85\end{array}$ & $\begin{array}{l}7.5 \\
2.40\end{array}$ & $\begin{array}{l}2.7 \\
0.11\end{array}$ \\
\hline & $\mathrm{T} 2$ & $\begin{array}{c}\text { mean } \\
\text { std }\end{array}$ & $\begin{array}{c}8.0 \\
0.08\end{array}$ & $\begin{array}{c}3.9 \\
0.44\end{array}$ & $\begin{array}{c}4.2 \\
1.27\end{array}$ & $\begin{array}{c}0.3 \\
0.06\end{array}$ & $\begin{array}{c}8.0 \\
1.06\end{array}$ & $\begin{array}{l}2750.0 \\
339.41\end{array}$ & $\begin{array}{l}98.0 \\
52.33\end{array}$ & $\begin{array}{l}8.5 \\
3.39\end{array}$ & $\begin{array}{l}29.8 \\
16.05\end{array}$ & $\begin{array}{l}17.4 \\
1.98\end{array}$ & $\begin{array}{l}12.3 \\
0.64\end{array}$ & $\begin{array}{l}1.9 \\
0.14\end{array}$ & $\begin{array}{l}1.7 \\
0.21\end{array}$ & $\begin{array}{l}5.8 \\
0.85\end{array}$ & $\begin{array}{l}9.7 \\
3.89\end{array}$ & $\begin{array}{l}2.4 \\
0.61\end{array}$ \\
\hline & T3 & $\begin{array}{c}\text { mean } \\
\text { std }\end{array}$ & $\begin{array}{c}8.0 \\
0.23\end{array}$ & $\begin{array}{c}3.7 \\
0.59\end{array}$ & $\begin{array}{c}3.2 \\
0.21\end{array}$ & $\begin{array}{c}0.2 \\
0.03\end{array}$ & $\begin{array}{c}7.7 \\
0.35\end{array}$ & $\begin{array}{c}2215.0 \\
1265.72\end{array}$ & $\begin{array}{l}90.5 \\
26.16\end{array}$ & $\begin{array}{l}8.8 \\
0.49\end{array}$ & $\begin{array}{l}20.6 \\
2.33\end{array}$ & $\begin{array}{l}18.1 \\
1.41\end{array}$ & $\begin{array}{l}10.3 \\
0.92\end{array}$ & $\begin{array}{l}1.8 \\
0.00\end{array}$ & $\begin{array}{l}1.6 \\
0.07\end{array}$ & $\begin{array}{l}5.3 \\
0.57\end{array}$ & $\begin{array}{l}9.2 \\
2.05\end{array}$ & $\begin{array}{l}1.9 \\
0.30\end{array}$ \\
\hline
\end{tabular}

\subsection{Mineral Composition}

Sorghum is an elemental raw material in animal feed because it supplies important minerals and micronutrients that are essential for optimal health, growth, and development. Animal nutrient requirements are dynamic and depend on specific individual needs and physiological states [26]. Table 4 shows the analyzed concentration of the Sorghum bicolor Payenne mineral elements. The statistical analysis presented a significant effect of treatment (a combination of water quality and irrigation systems) for the macronutrients analyzed in fodder, as shown in Table 3 using different numbers. Therefore, Ntot, Ptot, Ca, 
$\mathrm{Mg}$, and Na were significantly higher in the RW treatments (T1 and T2) than in the conventional water one (T3). This result is consistent with the higher contents of nitrate and $\mathrm{Na}$ observed in soils irrigated by RW. Also, although soils did not have higher orthophosphate content, higher P contents in leaves were obtained when irrigated with RW, as the same as obtained by Palacios-Diaz et al. [25]. These authors demonstrated that higher organic $\mathrm{P}$ and microbiological P in soils instead of higher orthophosphate $\mathrm{P}$ were obtained when irrigating using RW.

Table 4. Plant analysis. Total nitrogen (Ntot), total phosphorus (Ptot), potassium (K), calcium (Ca), magnesium (Mg), and sodium ( $\mathrm{Na})$, expressed as \%, and other elements (expressed as $\mathrm{mg} / \mathrm{kg})$ : boron $(\mathrm{B})$, copper $(\mathrm{Cu})$, iron (Fe), manganese (Mn), and zinc $(\mathrm{Zn})$ for sorghum at different harvest times (1, 2, 3, and 4) and for treatment (T1, T2, and T3) expressed as means and standard deviations.

\begin{tabular}{|c|c|c|c|c|c|c|c|c|c|c|c|c|c|}
\hline & \multirow[b]{2}{*}{ Harvest } & & \multicolumn{6}{|c|}{$\%$} & \multicolumn{5}{|c|}{$\mathrm{mg} / \mathrm{kg}$} \\
\hline & & & Ntot & Ptot & $\mathbf{K}$ & $\mathrm{Ca}$ & $\mathrm{Mg}$ & $\mathrm{Na}$ & B & $\mathrm{Cu}$ & $\mathrm{Fe}$ & Mn & $\mathrm{Zn}$ \\
\hline \multirow{8}{*}{$\mathrm{T} 1$} & \multirow{2}{*}{1 (90 days) } & means & $2.02^{\mathrm{c} 2}$ & 0.30 & $1.31^{\mathrm{ab} 1}$ & 0.36 & $0.35^{a b 12}$ & $0.14^{\mathrm{a} 1}$ & $13^{\mathrm{b} 1}$ & $6.33^{b 1}$ & $151^{\mathrm{ab} 1}$ & $62^{\mathrm{b} 1}$ & $19^{\mathrm{a} 1}$ \\
\hline & & std & 0.18 & 0.03 & 0.19 & 0.11 & 0.08 & 0.06 & 3.61 & 0.58 & 115.11 & 2.65 & 6.24 \\
\hline & \multirow[t]{2}{*}{2 (53 days) } & means & $1.6^{\mathrm{b} 2}$ & 0.33 & $1.21^{\mathrm{b} 1}$ & 0.15 & $0.24^{\text {a12 }}$ & $0.06^{\mathrm{a} 1}$ & $12.33^{\mathrm{b} 1}$ & $10.33^{\mathrm{c} 1}$ & $118.33^{b 1}$ & $44^{\mathrm{a} 1}$ & $30.67^{\mathrm{b} 1}$ \\
\hline & & std & 0.17 & 0.03 & 0.62 & 0.09 & 0.05 & 0.03 & 3.79 & 1.15 & 48.01 & 9.54 & 7.09 \\
\hline & \multirow{2}{*}{3 (100 days) } & means & $1.32 \mathrm{a} 2$ & 0.25 & $0.96^{\mathrm{a} 1}$ & 0.33 & $0.34^{\text {b12 }}$ & $0.19^{\mathrm{b} 1}$ & $12.67^{\mathrm{ab} 1}$ & $5.33^{a 1}$ & $84.67^{\mathrm{a} 1}$ & $48.7^{\mathrm{a} 1}$ & $23.33^{a b 1}$ \\
\hline & & std & 0.15 & 0.01 & 0.06 & 0.06 & 0.03 & 0.07 & 2.08 & 0.58 & 10.41 & 3.51 & 2.31 \\
\hline & \multirow{2}{*}{4 (48 days) } & means & $1.86^{\text {bc2 }}$ & 0.32 & $1.46^{\mathrm{b} 1}$ & 0.20 & $0.31^{\mathrm{b} 12}$ & $0.07^{\mathrm{a} 1}$ & $7.33^{\mathrm{a} 1}$ & $5.33^{a b 1}$ & $87.67^{a b 1}$ & $40.67^{\mathrm{a} 1}$ & $22.33^{a b 1}$ \\
\hline & & std & 0.13 & 0.02 & 0.08 & 0.05 & 0.03 & 0.02 & 1.53 & 0.58 & 25.03 & 6.66 & 2.52 \\
\hline \multirow{8}{*}{$\mathrm{T} 2$} & \multirow{2}{*}{1 (90 days) } & means & $2.01^{\mathrm{c} 2}$ & 0.29 & $1.31^{\mathrm{ab} 1}$ & 0.36 & $0.34^{\mathrm{ab} 2}$ & $0.11^{\mathrm{a} 1}$ & $15^{\mathrm{b} 1}$ & $6.67^{\mathrm{b} 1}$ & $61.67^{\text {ab1 }}$ & $69.67^{b 1}$ & $23.33^{a 1}$ \\
\hline & & std & 0.09 & 0.02 & 0.16 & 0.12 & 0.06 & 0.03 & 2.65 & 1.15 & 5.69 & 7.02 & 2.31 \\
\hline & \multirow{2}{*}{2 (53 days) } & means & $1.65^{\mathrm{b} 2}$ & 0.34 & $1.62 a^{b 1}$ & 0.30 & $0.33^{\mathrm{a} 2}$ & 0.09 a1 & $13.67^{\mathrm{b} 1}$ & $9.67^{\mathrm{cl}}$ & $182^{\mathrm{b} 1}$ & 50.67 a1 & $26.33^{b 1}$ \\
\hline & & std & 0.22 & 0.05 & 0.23 & 0.02 & 0.03 & 0.01 & 1.53 & 1.15 & 57.38 & 10.50 & 1.53 \\
\hline & \multirow[t]{2}{*}{3 (100 days) } & means & $1.34^{\mathrm{a} 2}$ & 0.27 & $1.08^{\mathrm{a} 1}$ & 0.39 & $0.37^{\mathrm{b} 2}$ & $0.15^{\mathrm{b} 1}$ & $10.67^{\mathrm{ab} 1}$ & $5^{\mathrm{a} 1}$ & $70.33^{\text {a1 }}$ & $50^{\mathrm{a} 1}$ & $24^{\mathrm{ab} 1}$ \\
\hline & & std & 0.05 & 0.03 & 0.17 & 0.05 & 0.01 & 0.02 & 2.89 & 0.00 & 6.51 & 11.79 & 2.65 \\
\hline & \multirow[t]{2}{*}{4 (48 days) } & means & $1.75^{\mathrm{bc} 2}$ & 0.31 & $1.45^{\mathrm{b} 1}$ & 0.26 & $0.35^{b 2}$ & $0.09^{\mathrm{a} 1}$ & $7.67^{\mathrm{a} 1}$ & $5^{\mathrm{ab} 1}$ & $131.33^{\mathrm{ab} 1}$ & $44^{\mathrm{a} 1}$ & $21.33^{\mathrm{ab} 1}$ \\
\hline & & std & 0.32 & 0.04 & 0.12 & 0.09 & 0.07 & 0.01 & 2.52 & 1.00 & 44.06 & 10.15 & 5.03 \\
\hline \multirow{8}{*}{ T3 } & \multirow{2}{*}{1 (90 days) } & means & $1.81^{\mathrm{c} 1}$ & 0.29 & $1.35^{\mathrm{ab} 1}$ & 0.24 & $0.26^{\mathrm{ab} 1}$ & $0.08^{\mathrm{a} 1}$ & $10.33^{\mathrm{b} 1}$ & $6^{\mathrm{b} 1}$ & $129^{a b 1}$ & $54.33^{\mathrm{b} 1}$ & $21.67^{\mathrm{a} 1}$ \\
\hline & & std & 0.32 & 0.06 & 0.16 & 0.01 & 0.03 & 0.01 & 2.08 & 1.73 & 16.64 & 12.34 & 1.53 \\
\hline & \multirow{2}{*}{2 (53 days) } & means & $1.37^{b 1}$ & 0.27 & $1.67^{\mathrm{b} 1}$ & 0.20 & $0.25^{\mathrm{a} 1}$ & $0.08^{a 1}$ & $10.33^{\mathrm{b} 1}$ & $9.33^{\mathrm{c} 1}$ & $108.33^{b 1}$ & $42.67^{\mathrm{a} 1}$ & $22.3^{\mathrm{b} 1}$ \\
\hline & & std & 0.17 & 0.01 & 0.03 & 0.03 & 0.01 & 0.02 & 3.51 & 0.58 & 18.01 & 15.04 & 2.08 \\
\hline & \multirow{2}{*}{3 (100 days) } & means & $1.10^{\mathrm{a} 1}$ & 0.23 & $1.21^{\mathrm{a} 1}$ & 0.23 & $0.3^{\mathrm{b} 1}$ & $0.17^{\mathrm{b} 1}$ & $9.33^{a b 1}$ & $5^{\mathrm{a} 1}$ & $68.33^{\mathrm{a} 1}$ & $46.7^{\mathrm{a} 1}$ & $24.67^{\mathrm{ab} 1}$ \\
\hline & & std & 0.13 & 0.02 & 0.29 & 0.02 & 0.01 & 0.11 & 1.15 & 0.00 & 4.51 & 6.66 & 2.52 \\
\hline & \multirow[t]{2}{*}{4 (48 days) } & means & $1.48^{\mathrm{bc} 1}$ & 0.29 & $1.41^{\mathrm{b} 1}$ & 0.27 & $0.35^{\mathrm{b} 1}$ & $0.07^{\mathrm{a} 1}$ & $8.67^{\mathrm{a} 1}$ & $5.67^{a b 1}$ & $128^{\mathrm{ab} 1}$ & $39.67^{\mathrm{a} 1}$ & $21.67^{\mathrm{ab} 1}$ \\
\hline & & std & 0.20 & 0.01 & 0.02 & 0.09 & 0.06 & 0.01 & 1.53 & 0.58 & 31.61 & 7.23 & 4.51 \\
\hline \multicolumn{3}{|c|}{ Sorghum Bicolor $^{1}$} & & 0.21 & 1.75 & 0.50 & 0.27 & 0.02 & & 9.00 & 392.00 & 65.00 & 31.00 \\
\hline \multicolumn{3}{|c|}{ Sorghum Sudanense $^{1}$} & & 0.24 & 2.57 & 0.67 & 0.31 & 0.03 & & 11.00 & 990.00 & 79.00 & 33.00 \\
\hline
\end{tabular}

Numbers with a different superscript letter: significant at the 0.05 level for harvest. Numbers with a different superscript number: significant at the 0.05 level for treatment. ${ }^{1}$ NRC-Nutrient Requirements of Dairy Cattle (2001).

This result coincides with [27], but these authors also reported a significant increment in K, probably due to the low adsorption capacity of the sandy loam soil that they indicated in their experiment. In our clay loam soil, $\mathrm{K}$ did not bring about a significant increase in plants. In fact, the nutrients added by RW play a key role in plant uptake, which would allow farmers to pay a lower fertilization cost because high yields of fodder crops need a large quantity of nutrients to be added to avoid soil depletion [28].

The Ntot, Ptot, $\mathrm{K}$, and Fe content significantly lowered with increased plant maturity (harvests 1 and 3, see Table 4, in which significant differences among harvests are shown using letters). Saini [29] reported that advanced plant age significantly lowered mineral 
matter. On the contrary, $\mathrm{Ca}, \mathrm{Na}$, and $\mathrm{Mn}$ had significantly higher contents in advanced mature plants. $\mathrm{Mg}, \mathrm{Cu}$, and $\mathrm{Zn}$ showed no clear tendency upon maturity. B contents increased over time in the plants irrigated with RW, probably as a response to soil accumulation. Therefore, in general terms, differences in mineral contents were related to water quality and the plant maturity stage.

The adequacy of a mineral supply from a particular compound ration can scarcely be predicted accurately enough using mineral composition tables for the principal feed constituents [30]. Mendoza-Grimón et al. [28] studied Pennisetum sp. (another high-yielding $\mathrm{C} 4$ grass), and obtained a $\mathrm{K} /(\mathrm{Ca}+\mathrm{Mg}$ ) ratio for all plant ages above 2.2 , which increases the possibility of inducing hypocalcemia. The same result was obtained in this study when irrigating with conventional water and for the less mature sorghum plants irrigated with treated water. Nevertheless, the mature sorghum plants of the first and third harvests irrigated with treated water had healthy values and lower ratios (1.8 and 1.4, respectively). The higher $\mathrm{Ca}$ and $\mathrm{Mg}$ contents in the plants irrigated with treated effluent can explain these results.

Another relevant ratio for animal feed is $\mathrm{Ca} / \mathrm{P}$. As pointed out by Harty [31], the optimum Ca:P ratio for cows, based on extensive research goes from 1.5:1 to 2:1. Besides, $\mathrm{Ca}$ and $\mathrm{P}$ requirements change with animal age and production stage. In our study, and for advanced mature plants (harvests 1 and 3), the obtained ratios are lower: around 1.2:1-1.4:1, and $0.82: 1-1: 1$ in the plants irrigated with the treated water and conventional water, respectively. For less mature plants (harvests 2 and 4), the ratios were around 0.45:1-0.83:1 and 0.74:1-0.93:1 for the plants irrigated with treated water and conventional water, respectively. Therefore, mineral contents vary less over time in those plants irrigated with conventional water, but also present a greater Ca:P imbalance at the same time.

The lower $\mathrm{Ca}, \mathrm{P}$, and $\mathrm{K}$ values and microelement values for the Payene variety herein cultivated were obtained by comparing Soghum bicolor and Sorghum sudanense [32]. On the contrary, the $\mathrm{Na}$ measured in these plants was higher than in Soghum bicolor, but lower than in Sorghum sudanense. Khalil et al. [33], studied mineral contents in Sorghum bicolor, and reported that mineral elements $\mathrm{P}, \mathrm{K}, \mathrm{Mg}$, and $\mathrm{Ca}$ were the most abundant, while Fe, $\mathrm{Na}, \mathrm{Mn}$, and $\mathrm{Zn}$ appeared in small quantities. Our experiment partially coincided, and the most abundant were $\mathrm{K}, \mathrm{Mg}$, $\mathrm{P}$, and $\mathrm{Ca}$, with similar $\mathrm{Na}$ and Fe contents $(0.11 \%)$, followed by $\mathrm{Mn}, \mathrm{Zn}, \mathrm{B}$, and $\mathrm{Cu}$. As previously mentioned, the most abundant minerals (except for $\mathrm{K}$, which is a highly adsorbed nutrient by soil, Table 3) in the plants irrigated with treated water were significantly higher than when conventional water was employed. Therefore, the reuse of treated water allowed the fertilization cost to lower by avoiding soil depletion. The presented results coincide with Gerrano et al. [34], who mentioned that the mineral element contents in sorghum varied due to genotypic and environmental influences, and because of genotypes by environment interactions, which suggest the need to carry out experimental studies under local conditions.

As indicated by Palacios et al. [3], neither nutrient deficiencies nor important imbalances were observed, which suggests the contribution of nutrients from irrigation water, and probably from residual soil forms. Macrominerals in livestock production systems are either added to the total mixed ratios or presented in blocks to overcome the mineral imbalance problems observed in fodder plants. Although it is not known how ruminants self-select mineral supplements, whose $\mathrm{Ca}$ and $\mathrm{P}$ concentrations vary according to the physiological state [26], these authors demonstrated that lambs were able to discriminate among minerals, and some groups modify their preferences given the influence by mineral needs which, in turn, contributes to rectify mineral imbalances.

Our results also coincide with those of Ali et al. [35], who mentioned that reclaimed water irrigation does not increase the mineral concentrations of either macro- or microelement in plants to hazardous limits according to established standards, and can be used safely for crop irrigation purpose. Therefore, in this study, the recycled nutrients added by treated water contribute to circular economy. 


\subsection{NDF and ADF Composition}

ADF and NDF represent the fibrous portions of plant material. The digestibility and energy available to animals from forage are influenced by these parameters [36]. Tables 5 and 6 show the NDF and ADF contents to be affected by treatment and maturity upon harvest. No statistical differences in NDF and ADF were observed per treatment. Albeit not significantly so, a tendency to obtain lower NDF values (Table 5) in the plants irrigated using RW was observed, except at the second harvest. These results coincide with those obtained by other authors, who mentioned that irrigation with sewage water significantly lowered NDF \% [37]. Galavi et al. [27] mentioned that reduced stem fiber could be due to not only $\mathrm{N}$ added by treated water, but also to solution carbohydrate enlargement and, eventually, the NDF results decrease upon forage. Significantly higher mean ADF and NDF values were obtained by Janhi et al. [38] in forage sorghum that was not supplemented with $\mathrm{N}$ compared to treatments with added $\mathrm{N}$.

Table 5. The Neutral Detergent Fiber (NDF) content in sorghum affected by both the irrigation system and water quality (Treatment) for different harvest periods.

\begin{tabular}{|c|c|c|c|c|}
\hline \multirow[b]{2}{*}{ Harvest } & & \multicolumn{3}{|c|}{ NDF\% } \\
\hline & & T1 & $\mathrm{T} 2$ & T3 \\
\hline \multirow{2}{*}{1} & means & $53.81^{a}$ & $50.38^{a}$ & $56.23^{a}$ \\
\hline & std & 2.10 & 8.85 & 2.68 \\
\hline \multirow{2}{*}{2} & means & $61.42^{b}$ & $65.26^{\mathrm{b}}$ & $62.31^{\mathrm{b}}$ \\
\hline & std & 0.67 & 1.47 & 1.38 \\
\hline \multirow{2}{*}{3} & means & $61.06^{b c}$ & $61.42^{b c}$ & $61.95^{b c}$ \\
\hline & std & 0.58 & 2.76 & 0.85 \\
\hline \multirow{2}{*}{4} & means & $59.43^{c}$ & $59.08^{c}$ & $61.53^{c}$ \\
\hline & std & 1.54 & 1.77 & 1.30 \\
\hline \multicolumn{2}{|c|}{ Sorghum bicolor ${ }^{1}$} & & 59 & \\
\hline \multicolumn{2}{|c|}{ Sorghum sudanense $^{1}$} & & 63.3 & \\
\hline
\end{tabular}

Numbers with a different letter superscript: significant at the 0.05 level for time numbers with a different number superscript: significant at the 0.05 level for treatment. ${ }^{1}$ NRC - Nutrient Requirements of Dairy Cattle (2001)

Table 6. The acid Detergent Fiber (ADF) content in sorghum is affected by irrigation system and water quality (Treatment) and different harvest periods.

\begin{tabular}{|c|c|c|c|c|}
\hline \multirow[b]{2}{*}{ Harvest } & & \multicolumn{3}{|c|}{$\mathrm{ADF} \%$} \\
\hline & & T1 & $\mathbf{T} 2$ & T3 \\
\hline \multirow{2}{*}{1} & means & $33.90^{\mathrm{a} 1}$ & $29.542^{a 1}$ & $29.63^{\mathrm{a} 1}$ \\
\hline & std & 9.58 & 5.29 & 4.98 \\
\hline \multirow{2}{*}{2} & means & $32.70^{b 1}$ & $36.14^{b 1}$ & $36.85^{b 1}$ \\
\hline & std & 1.42 & 3.79 & 7.31 \\
\hline \multirow{2}{*}{3} & means & $30.40^{\mathrm{ab} 1}$ & $33.32^{a b 1}$ & $34.50^{a b 1}$ \\
\hline & std & 6.26 & 2.10 & 1.49 \\
\hline \multirow{2}{*}{4} & means & $32.33^{a b 1}$ & $31.67^{\mathrm{ab} 1}$ & $33.41^{\mathrm{ab} 1}$ \\
\hline & std & 0.42 & 2.15 & 0.79 \\
\hline \multicolumn{2}{|c|}{ Sorghum bicolor ${ }^{1}$} & \multicolumn{3}{|c|}{36.659} \\
\hline \multicolumn{2}{|c|}{ Sorghum sudanense ${ }^{1}$} & \multicolumn{3}{|c|}{40.7} \\
\hline
\end{tabular}

Numbers with a different letter superscript: significant at the 0.05 level for time numbers with a different number superscript: significant at the 0.05 level for treatment. ${ }^{1}$ NRC - Nutrient Requirements of Dairy Cattle (2001). 
The harvest effect was significant for both the NDF and ADF contents. The NFD contents increased with advanced maturity. The maximum and minimum NDF contents were analyzed for the second and first harvests (62.31 and 50.38, respectively). This result coincides with Lemerle et al. [39], who reported increased NDF with delayed maize harvesting.

Lyons et al. [40] studied ADF values in the boot, flower, milk, and soft dough stages, and noted a progressive increment in ADF with maturity. On the contrary and albeit not significantly (Table 6), the lowest ADF $(30.40 \% \pm 6.26)$ was obtained in T1 (treatment reusing RW and SDI) and at the third harvest (110 days between harvests). As RW adds a considerable amount of $\mathrm{N}$, this parameter is probably related to the decrease in cellulose and hemicellulose observed in fodder. This finding has been reported by Vuckovic et al. [41] when applying nitrogen fertilizer to pasture grasses.

Our results coincide with the values reported by Atis et al. [42] and Singh and Shukla [43] when harvesting sorghum in the milking stage, with a mean concentration of $65.80 \%$ NDF and $38.22 \%$ ADF. Generally, the NDF and ADF contents tended to lower with delayed harvest time [42]. When we compared the results obtained for sorghum var Payenne with the aforementioned NCR values [32], our study gave higher values for NDF than for Sorghum bicolor, but lower ones than those obtained for Sorghum sudanense. Regarding ADF, sorghum var Payenne had lower values compared to Sorghum bicolor and sudanense.

When considering the fiber values obtained during this experiment, all the values met the No. 2 and 3 standards and the Prime and No. 1 standard [44] for NDF and ADF, respectively. Therefore, good quality forage was produced to cover animal nutrient requirements. If treated water reuse to irrigate fodder crops could be generalized in Cape Verde, it would be possible to partially replace forage importation.

\section{Conclusions}

The presented pilot project demonstrated the feasibility of producing fodder crops by reusing the treated water produced by a wastewater treatment plant adapted to a rural village. Good quality forage in nutrients and fiber terms was obtained reusing water by a subsurface drip irrigation, a safety system which minimizes sanitary risks by avoiding contact between treated water and aerial plant parts. The proposed reuse helps to avoid the conventional water competence and permit the reuse of nutrients added by RW. In general terms, the differences in mineral contents are related to water quality and the plant maturity stage. As ruminants self-select mineral supplements from mineral blocks according to physiological state, they are able to rectify the slight mineral imbalances detected in forages produced by this pilot project. Albeit not significant, a tendency to obtain lower NDF values in the plants irrigated using RW was observed, and is probably related to the $\mathrm{N}$ provided by treated water. If generalized to other small villages, the proposed solution would allow a partial reduction in forage importation, which would improve food sovereignty and farmers' profitability by increasing resilience against climate change. Further studies should analyze other environmental, economic, and social issues to guarantee the sustainability of the proposed reuse system.

Author Contributions: M.d.P.P.-D., V.M.-G., and J.M.H.-M. had the original idea for this study. V.M.G., M.d.P.P.-D., and R.A. were responsible for data collection in the field. J.R.F.-V., R.A., and V.M.-G. were responsible for carrying out the analyses, while M.d.P.P.-D. and V.M.-G. were responsible for data cleaning and analyses. V.M.-G., J.M.H.-M., and M.d.P.P.-D. drafted the manuscript, which was revised by all the authors. All authors have read and agreed to the published version of the manuscript.

Funding: This research was funded by the Interreg MAC 2104-2020 Program (ADAPTaRES Proyect: http:/ /adaptares.com/es/). (accessed on 10 March 2021).

Institutional Review Board Statement: Not applicable.

Informed Consent Statement: Not applicable. 
Data Availability Statement: The data presented in this study are available on request from the corresponding author.

Acknowledgments: We also appreciate the cooperation of the Laboratorio Agroalimentario del Cabildo de Gran Canaria, ITC, SISTEMA INGENIERIA, INDUS, and INIDA.

Conflicts of Interest: The authors declare no conflict of interest.

\section{References}

1. Mendoza-Grimón, V.; Hernández-Moreno, J.M.; Palacios-Díaz, M.D.P. Improving water use in fodder production. Water 2015, 7, 2612-2621. [CrossRef]

2. Palacios-Díaz, M.P.; Mendoza-Grimón, V.; Fernández-Vera, J.R.; Hernández-Moreno, J.M. Effects of defoliation and nitrogen uptake on forage nutritive values of Pennisetum sp. J. Anim. Plant Sci. 2013, 23, 566-574.

3. Palacios, M.P.; Mendoza-Grimón, V.; Fernández, F.; Fernández-Vera, J.R.; Hernández-Moreno, J.M. Sustainable Reclaimed Water Management by Subsurface Drip Irrigation System: A study case for forage production. Water Pract. Technol. 2008, 3. [CrossRef]

4. Mendoza-Grimón, V.; Fernández-Vera, J.R.; Silva, G.D.; Semedo-Varela, A.; Palacios-Díaz, M.D.P. Cape Verde (West Africa) Successful Water Reuse Pilot Project: A Sustainable Way for Increasing Food Production in a Climate Change Scenario. Water 2021, 13, 160. [CrossRef]

5. Winpenny, J.; Heinz, I.; Koo-Oshima, S. Reutilización del Agua en Agricultura: Beneficios Para Todos? FAO Informe sobre Temas hídricos: Rome, Italy, 2013; p. 35.

6. Available online: https://www.ifad.org/en/web/operations/country/id/cape_verde (accessed on 20 March 2021).

7. ANAS. Estação de Tratamento de Águas Residuais "ETAR" Cabo Verde. Estudo e Caracterização; Departamento de Gestão de Recursos Hídricos e Saneamento "DGRHS", Agência Nacional de Água e Saneamento "ANAS": June 2016. Available online: www.http: / / anas.gov.cv (accessed on 10 January 2021).

8. SDG Cabo Verde. Sustainable Develpoment Goal. Voluntary National Report on the Implementation of the 2023 Agenda for Sustainable Development. 2018. Available online: https://sustainabledevelopment.un.org (accessed on 15 March 2021).

9. INE.CV. 2018. Available online: http://ine.cv/estatisticas-por-tema/ (accessed on 15 March 2021).

10. Satyavathi, C.T.; Solanki, R.; Kakani, R.; Bharadwaj, C.; Singhal, T.; Padaria, J.; Khandelwal, V.; Srivastava, R.; Tomar, R.S.; Iqubal, M.A. Genomics assisted breeding for abiotic stress tolerance in Millets. In Genomics Assisted Breeding of Crops for Abiotic Stress Tolerance; Springer: New York, NY, USA, 2019; pp. 241-255.

11. Staggenborg, S.; Dhuyvetter, K.C.; Gordon, W.B. Grain sorghum and corn comparisons: Yield, economic and environmental responses. Agron. J. 2008, 100, 1600-1604. [CrossRef]

12. Bolaños, A.E.D.; Emile, J.-C. Distancia entre surcos en el rendimiento y calidad de la materia seca de maíz y de sorgo. Rev. Mex. Cienc. Реси. 2011, 2, 299-312.

13. Texas Cooperative Extension and Texas Agricultural Experiment Station. Bean and McCollum. Summary of Six Years of Forage Sorghum Variety Trials. Pub. SCS-2006-04; Texas Cooperative Extension and Texas Agricultural Experiment Station: College Station, TX, USA, 2006.

14. McCuistion, K.C.; McCollum, F.T.; Bean, B.W.; Rowland, M.W. Observations on nutritional value of forage sorghums for silage. In Proceedings of the Plains Nutrition Council Spring Conference, San Antonio, TX, USA, 15-16 April $2004 ;$ p. 105.

15. Oliver, A.L.; Grant, R.J.; Pedersen, J.F.; O'Rear, J. Comparison of brown midrib-6 and -18 forage sorghum with conventional sorghum and corn silage in diets of lactating dairy cows. J. Dairy Sci. 2004, 87, 637-644. [CrossRef]

16. Pedersen, J.F.; Gorz, H.J.; Haskins, F.A.; Ross, W.M. Variability for quality and agronomic traits in forage sorghum hybrids. Crop Sci. 1982, 22, 853-856. [CrossRef]

17. ISRA. Fiches Techniques. In Institut Sénégalais de Recherches AgricolesFiches Variétales Niébé \& Sorgho; ISRA: Dakar, Senegal, 2015; Volume 8, ISSN 0850-9980.

18. Ball, D.M.; Collins, M.; Lacefield, G.D.; Martin, N.P.; Mertens, D.A.; Olson, K.A.; Putnam, D.H.; Under-sander, D.J.; Wolf, M.W. Understanding Forage Quality. American Farm Bureau Federation Publication 1-01; American Farm Bureau Federation: Park Ridge, IL, USA, 2001.

19. Tasie, M.M.; Gebreyes, B.G. Characterization of Nutritional, Antinutritional, and Mineral Contents of Thirty-Five Sorghum Varieties Grown in Ethiopia. Int. J. Food Sci. 2020, 2020. [CrossRef]

20. Smith, M. CROPWAT: A Computer Program for Irrigation Planning and Management; Food and Agriculture Organization of the United Nations, Ed.; FAO Irrigation and Drainage Paper 46; Food and Agriculture Organization of the United Nations: Rome, Italy, 1992; ISBN 29251031061. Available online: http:/ / www.fao.org/land-water/databases-and-software/cropwat/es/ (accessed on 10 March 2021).

21. Soil Survey Staff. Keys to Soil Taxonomy, 8th ed.; 1998-NRCS.; U.S.D.A.: Lincoln, NE, USA, 1998.

22. IUSS Working Group WRB. World Reference Base for Soil Resources 2014, Update 2015. International Soil Classification System for Naming Soils and Creating Legends for Soil Maps. World Soil Resources Reports No. 106; FAO: Rome, Italy, 2015.

23. Olsen, S.R.; Sommers, L.E. Phosphorus. In Methods of Soil Analysis. Part2. Chemical and Microbiological Properties, Agronomy Monograph 9.2; Miller, R.H., Keeney, E.R., Eds.; America Society Agronomy, Inc.: Madison, WI, USA; Soil Science of America, Inc.: Madison, WI, USA, 1982; pp. 1035-1049. 
24. Van Soest, P.J.; Robertson, J.B.; Lewis, B.A. Methods for Dietary Fiber. Neutral Detergent Fiber. and Nonstarch Polysaccharides in Relation to Animal Nutrition. J. Dairy Sci. 1991, 74, 3583-3597. [CrossRef]

25. Palacios-Díaz, M.P.; Mendoza-Grimón, V.; Fernández-Vera, J.R.; Rodríguez-Rodríguez, F.; Tejedor-Junco, M.T.; HernándezMoreno, J.M. Subsurface drip irrigation and reclaimed water quality effects on phosphorus and salinity distribution and forage production. Agric. Water Manag. 2009, 96, 1659-1666. [CrossRef]

26. Perderna, M.; Mereu, A.; Cromer Eand Villalba, J.J. Preference for inorganic sources of calcium and phosphorus by sheep as a function of need. Anim. Sci. J. 2020. 91, 13460.

27. Galavi, M.; Jalali, A.; Mousavi, S.R.; Galavi, H. Effect of treated municipal wastewater on forage yield, quantitative and qualitative properties of sorghum (Sorghum bicolor Speed feed). Asian J. Plant Sci. 2009, 8, 489-494. [CrossRef]

28. Mendoza-Grimón, V.; Fernández-Vera, J.R.; Hernández Moreno, J.M.; Palacios-Díaz, M.P. Mineral balance and absorption from soil of Pennisetum sp at different stages. Int. J. Environ. Agric. Res. 2016, 2, 29-35.

29. Saini, A. Forage quality of Sorghum (Sorghum bicolor) as influenced by Irrigation, nitrogen levels and harvesting stage. Indian J. Sci. Res. 2012, 3, 67-72.

30. Suttle. Natural sources on minera. In Mineral Nutrition of Livestock, 4th ed.; British Library: London, UK, 2010.

31. Harty, A. Cow Mineral Nutrition: Macro Minerals and Their Importance. Available online: https://extension.sdstate.edu/cowmineral-nutrition-macro-minerals-and-their-importance (accessed on 10 June 2020).

32. NRC. Nutrient Requirements of Dairy Cattle, 7th ed.; Clark, J.H., Beede, D.K., Erdman, R.A., Goff, J.P., Grummer, R.R., Linn, J.G., Pell, A.N., Schwab, C.G., Tomkins, T., Varga, G.A., et al., Eds.; National Academy Press: Washington, DC, USA, 2001.

33. Khalil, J.K.; Sawaya, W.N.; Al Mohammed, H.M. Chemical composition and nutritional quality of sorghum flour and bread. Qual. Plant Foods Hum. Nutr. 1984, 34, 141-150. [CrossRef]

34. Gerrano, A.S.; Labuschagne, M.T.; van Biljon, A.; Shargie, N.G. Quantification of Mineral Composition and Total Protein Content in Sorghum [Sorghum Bicolor (L.) Moench] Genotypes. Cereal Res. Commun. 2016, 44, 272-285. [CrossRef]

35. Al-Jaloud, A.A.; Hussain, G.; Al-Saati, A.J.; Karimulla, S. Effect of wastewater irrigation on mineral com-position of corn and sorghum plants in a pot experiment. J. Plant Nutr. 1995, 18, 1677-1692. [CrossRef]

36. Bean, B.W.; Baumhardt, R.L.; McCollum, F.T.; McCuistion, K.C. Comparison of sorghum classes for grain and forage yield and forage nutritive value. Field Crops Res. 2013, 142, 20-26. [CrossRef]

37. Soni, P.G.; Yadav, R.K.; Kumar, A.; Yadav, G.; Kumar, G.; Yadav, T. Effect of Domestic Wastewater and Irrigation Schedules on Quality of Fodder Sorghum. J. Soil Salin. Water Qual. 2016, 8, 173-179.

38. Janhi, K.; Matshaya, Z.; Chiduza, C.; Muzangwa, L. Clipping Forage Sorghum Twice and Nitrogen Topdressing Offer an Option for Dual-Purpose Use for Cover Cropping and Fodder in Mixed Crop/Livestock Farming Systems. Agronomy 2020, 10, 17. [CrossRef]

39. Lemerle, C.; Etheridge, M.O.; Trigg, T.E. The effect of stages of maturity on digestibility and chemical composition of maize. In Proceedings of the Symposium at the University of New England, 24-27 November 1985.

40. Lyons, S.E.; Ketterings, Q.M.; Godwin, G.S.; Cherney, D.J.; Cherney, J.H.; Van Amburgh, M.E.; Meisinger, J.J.; Kilcer, T.F. Optimal harvest timing for brown midrib forage sorghum yield, nutritive value, and ration performance. J. Dairy Sci. 2019, 102, 7134-7149. [CrossRef] [PubMed]

41. Vuckovic, S.; Cupina, B.; Simic, A.; Prodanovic, S.; Zivanovic, T. Effect of nitrogen fertilization and under sowing on yield and qualitative of cynosuretumcristati type meadows in hilly-mountains grasslands in Serbia. J. Cent. Eur. Agric. 2005, 6, 509-514.

42. Atis, I.; Konuskan, O.; Duru, M.; Gozubenli, H.; Yilmaz, S. Effect of harvesting time on yield, composition and forage quality of some forage sorghum cultivars. Int. J. Agric. Biol. 2012, 14, 879-886.

43. Singh, S.; Shukla, G.P. Genetic diversity in the nutritive value of dual purpose sorghum hybrids. Anim. Nutr. Feed Technol. 2010, 10, 93-100.

44. Blezinger, S. Developing Sound Forage Analyses a Program. 1999. Available online: http://www.cattletoday.com/archive/1999 /September/Cattle_Today20.shtml (accessed on 22 March 2021). 